\title{
Charge Orderings and Phase Separations in Itinerant Fermion Systems at Half Filling
}

\author{
W.R. Czart*, P.R. Grzybowski, M. Nogala and S. Robaszkiewicz \\ Faculty of Physics, A. Mickiewicz University, Umultowska 85, 61-614 Poznań, Poland
}

\begin{abstract}
We analyse the ground state phase diagrams of the charge orderings in narrow band materials using two effective models: (1) the spinless fermion model $(t-W)$ with repulsive intersite interaction $\left(W_{i j}>0\right)$ and $(2)$ the molecular crystal model with the coupling of electrons to intramolecular (crystal field) vibrations. We present results for the case of half filled bands for $d=2$ square lattice. The calculations are performed within the (broken symmetry) Hartree-Fock approximation. The study takes into consideration the effects of frustrating next-nearest-neighbour hopping $\left(t_{2}\right)$ on the charge ordered states in these systems. We focus on the two cases: (i) homogeneous phases and phase separations involving checkerboard charge ordering with the nesting vector $Q=(\pi, \pi)$ only and (ii) homogeneous phases and phase separations involving two types of charge ordering: (a) checkerboard charge ordering with the nesting vector $Q=(\pi, \pi)$, and (b) collinear (CL) charge ordering with $Q=(0, \pi)$ or $Q=(\pi, 0)$.
\end{abstract}

PACS: 71.10.Fd, 71.30. $+\mathrm{h}$, 71.45.Lr, 64.75.Gh

\section{Introduction}

Two main mechanisms are invoked in explaining charge orderings ( $\mathrm{CO})$ phenomena: electron correlations and lattice distortion ([1-8] and references therein). In previous papers $[3,8]$ we analysed $(1)$ one of the paradigmatic models of correlated electrons - the so-called $t-W$ model of spinless fermions with repulsive intersite interaction $W[3]$ and (2) a simple paradigmatic model of electron-lattice interactions - the molecular crystal (MC) model in the static limit, with electrons coupled to intramolecular (crystal field) vibrations [8]. So far our analysis has been concentrated on the problem of phase separations (PS) involving checkerboard (CB) CO only.

In this contribution we have extended our previous works by considering collinear (CL) charge orderings. We present results at half filling for $\mathrm{MC}$ and $t-W$ models and compare them for two cases: (i) PS involving CB CO only and (ii) PS involving both CB and CL CO.

Collinear charge orderings have been reported in the RPA calculations of collective modes in the extended attractive Hubbard model [9]. Infinite-dimensional generalizations of collinear orderings have been shown to be stable analogs of incommensurate phases in the $t-W$ model on $d=\infty$ hypercubic lattice [10]. Here we present, for the first time, an overview of the existence of CL CO on the ground state (GS) phase diagrams of the $t-W$ model and the MC model on $d=2$ square lattice, in a presence of next nearest-neighbour hopping.

The model Hamiltonians have the following form: (1) the $t-W$ model with intersite density interaction $[3,4]$ :

\footnotetext{
* corresponding author; e-mail: czart@amu.edu.pl
}

$$
\begin{aligned}
& \hat{H}=\sum_{i j} t_{i j} c_{i}^{+} c_{j}+\frac{1}{2} \sum_{i j} W_{i j} n_{i} n_{j}-\mu \sum_{i} n_{i}, \\
& n_{\mathrm{s}}=\frac{N_{\mathrm{s}}}{N}=\frac{1}{N} \sum_{i}\left\langle n_{i}\right\rangle, \quad 0<n_{\mathrm{s}}<1 .
\end{aligned}
$$

(2) the MC model $[4,8]$ :

$$
\begin{aligned}
\hat{H} & =\sum_{i j \sigma} t_{i j} c_{i \sigma}^{+} c_{j \sigma}+\frac{1}{\sqrt{N}} \sum_{i q v} A_{q v}^{E} \mathrm{e}^{\mathrm{i} \boldsymbol{q} \cdot \boldsymbol{R}_{i}} n_{i} \hat{\varphi}_{q v} \\
& +\sum_{q v} \Omega_{q v} b_{q v}^{+} b_{q v}-(\mu-E) \sum_{i \sigma} n_{i \sigma}, \\
n_{E} & =\frac{N_{E}}{N}=\frac{1}{N} \sum_{i \sigma}\left\langle n_{i \sigma}\right\rangle, \quad 0<n_{E}<2 .
\end{aligned}
$$

In both models $t_{i j}$ are the single particle hopping integrals (between the nearest neighbours $t$ and the next nearest neighbours $\left.t_{2}\right), \mu$ - the chemical potential.

In the model (1) $c_{i}^{+}\left(c_{i}\right)$ are the creation (annihilation) operators for spinless fermions on site $i, n_{i}=c_{i}^{+} c_{i}, W_{i j}$ are the intersite density-density interactions, assumed to be repulsive $\left(W_{i j}>0\right)$, and restricted to nearest neighbours.

In the model (2) $c_{i \sigma}^{+}\left(c_{i \sigma}\right)$ are the creation (annihilation) operators for fermions on site $i, n_{i \sigma}=c_{i \sigma}^{+} c_{i \sigma}, A_{q v}^{E}$ terms describe the coupling of electrons to various types of intramolecular (or cation-ligand) vibrations via modulation of the molecular (crystal field) energy $E, \Omega_{q v}$ are the phonon branches, arising from these intramolecular (cation-ligand) vibrations [4], $b_{q v}^{(+)}$are phonon operators of the $v$-th phonon branch, $\hat{\varphi}_{q v}=b_{q v}+b_{q v}^{+}$. We restrict considerations to the static limit and assume that the macroscopic distortions are caused by phonon modes with $q=\boldsymbol{Q}(\boldsymbol{Q}$ - half the smallest reciprocal lattice vector). Therefore we put [4] 


$$
\begin{aligned}
& b_{q v}^{(+)} \rightarrow\left\langle b_{q v}^{(+)}\right\rangle=\frac{1}{2} \sqrt{N}\left\langle\varphi_{q v}\right\rangle \delta_{|\boldsymbol{q}|, \boldsymbol{Q}}, \\
& \hat{\varphi}_{q v} \rightarrow \sqrt{N} \varphi_{q v} \delta_{|\boldsymbol{q}|, \boldsymbol{Q}},
\end{aligned}
$$

neglecting all phonons with $\boldsymbol{q} \neq \boldsymbol{Q}$ and determining the classical field $\varphi_{Q v}$ by minimizing of the free energy (for macroscopic distortion the phonon amplitudes may be treated classically i.e. the large quantum number correspondence-principle).

We have performed extensive studies of both models within broken symmetry Hartree-Fock approximation for arbitrary particle concentrations $\left(n_{\mathrm{s}}, n_{E}\right)$. Below we only shortly summarize the main findings obtained at half-filling and $d=2$ lattice presenting selected phase diagrams which have been evaluated at $T=0$.

\section{Results and discussion}

We use the following notation (for $d=2$ SQ lattice): $W_{0}=2 d W / t$, for the model (1) and $G=G_{Q}^{E} / t$, where $G_{Q}^{E}=\sum_{v}\left(4\left(A_{Q v}^{E}\right)^{2} / \Omega_{Q v}\right)$, for the model (2), $b=t_{2} / t$.

In the model (1) the $\mathrm{CO}$ phases are characterized by the electron CO parameter: $\Delta_{Q}^{s}=\frac{1}{N} \sum_{i}\left\langle n_{i}\right\rangle \mathrm{e}^{\mathrm{i} \boldsymbol{Q} \cdot \boldsymbol{R}_{i}} \neq 0$, where $Q=(\pi, \pi)$ for $\mathrm{CB} \mathrm{CO}$ and $Q=(\pi, 0)$ or $Q=$ $(0, \pi)$ for CL CO, whereas in the model (2) by: $\Delta_{Q}^{e}=$ $\frac{1}{N} \sum_{i \sigma}\left\langle n_{i \sigma}\right\rangle \mathrm{e}^{\mathrm{i} \boldsymbol{Q} \cdot \boldsymbol{R}_{i}} \neq 0$ and simultaneously by the static internal distortions: $\varphi_{Q v}=-\left(2 A_{Q v}^{E} \Delta_{Q}^{\mathrm{e}}\right) /\left(\Omega_{Q v}\right) \neq 0$.

\subsection{The phase diagrams for the case of $P S$ involving $C B C O$ only}

In the absence of frustration $\left(t_{2}=0\right)$ at half-filling (i.e. for $n_{\mathrm{s}}=0.5$ for the $t-W$ model and $n_{E}=1$ in case of the $\mathrm{MC}$ model) and considering the phase separation involving CB CO only [3], the ground state (GS) of both models for $d \geq 2$ hypercubic lattices is homogeneous $\mathrm{CB}$ CO for any interaction strength $W_{0}, G>0$ (see Figs. 1a,b, 2a,b).

The next-nearest-neighbour hopping $t_{2}$ breaks the electron-hole symmetry of the systems considered and for both models it suppresses the perfect nesting instability towards CB CO phases at weak interactions strength $W_{0}($ or $G)$ at half-filling.

The critical interactions $W_{\mathrm{c} 0}$ (or $G_{\mathrm{c}}$ ) necessary to stabilize the $\mathrm{CB}$ CO state decrease rather slowly with decreasing $t_{2}$ for small $b\left[\left(W_{\mathrm{c} 0}\right)^{-1} \sim\left(G_{\mathrm{c}}\right)^{-1} \sim|\ln (b)|\right]$.

In the case of PS involving $\mathrm{CB} \mathrm{CO}$ only, the GS diagrams for both models consist of three states (cf. Fig. 1a, 2a). The first is the non-ordered metal (NO) if the interaction parameter (IP) is smaller than a critical value for a given $t_{2}$. If we increase IP the system settles in the phase separated state: $\mathrm{CB} \mathrm{CO} / \mathrm{NO}$. The transitions at the critical value of the IP for both models are of the 1 st order for any $t_{2}$, and the critical value increases with the increase of $t_{2}$. Above enough high value of IP the system enters the homogeneous CB CO state. Summarising, for $t_{2} \neq 0$ the increasing of interaction parameter yields a sequence of two 1st order transitions $\mathrm{NO} \rightarrow \mathrm{PS}(\mathrm{CB} / \mathrm{NO}) \rightarrow \mathrm{CB} \mathrm{CO}$.

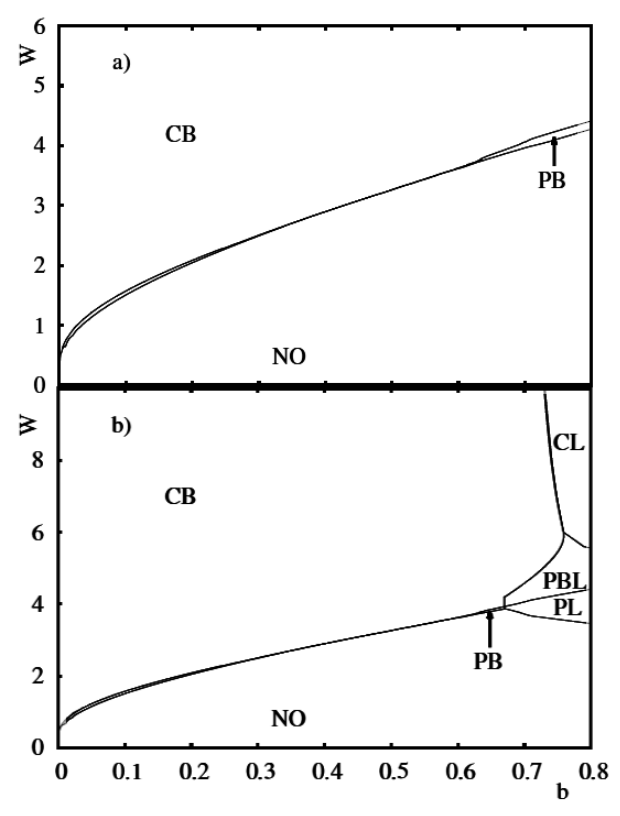

Fig. 1. Ground state phase diagrams for the $t-W$ model at half filling $\left(n_{\mathrm{s}}=0.5\right)$ as a function of $b$ for the two cases: (a) taking into account $\mathrm{CB} C \mathrm{CO}$ only (the phase separated region is marked $\mathrm{PB}$ for $\mathrm{PS}(\mathrm{CB} / \mathrm{NO}))$, (b) taking into account $\mathrm{CL}$ and $\mathrm{CB}$ CO (the phase separated regions involving $\mathrm{CL}$ are marked PL for PS(CL/NO), and PBL for PS(CB/CL)). $d=2$ SQ lattice.

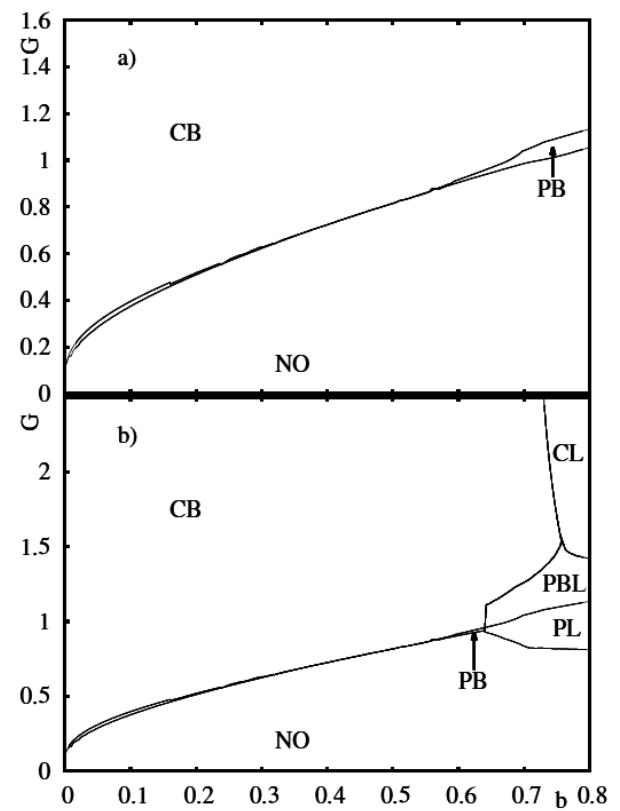

Fig. 2. Ground state phase diagrams for the MC model in the static limit at half filling $\left(n_{E}=1\right)$ as a function of $b$ for the two cases: (a) taking into account CB CO only, (b) taking into account CL and CB CO. $d=2 \mathrm{SQ}$ lattice. Denotations as in Fig. 1. 
For large frustration the area of a phase diagram occupied by the $\mathrm{PS}(\mathrm{CB} / \mathrm{NO})$ state is relatively wide, while for smaller values of $b$ the area of phase separation is quite narrow.

\subsection{The phase diagrams taking into account both $C L$ and $C B C O$}

In the absence of frustration $\left(t_{2}=0\right)$ when considering possibility of existence of both types of $\mathrm{CO}$, the ground state of both models at half-filling for $d \geq 2$ hypercubic lattices is found to be homogeneous $\mathrm{CB}$ CO for any interaction strength $G, W_{0}>0$ as in the case $A$.

An increase of $t_{2}$ changes the perfect nesting instability towards CB CO, into the instability towards CL CO. For large $t_{2}$ values, the CL CO phase takes over the CB CO phase. The frustration introduced by any $t_{2} \neq 0$ results in the finite value of $W_{0}(G)$ below which no CO phase occurs for $n_{\mathrm{s}}=0.5\left(n_{E}=1\right)$ and system remains in the NO metallic phase.

In the presence of large frustration the GS phase diagrams are considerably changed comparing to the case of taking into account $\mathrm{CB}$ CO only (cf. Figs. 1, 2a with Figs. 1, 2b). At $T=0$ for both models at half-filling and $t_{2} \neq 0$ one can observe several various types of behaviour, depending on interaction parameter, and $b$ : (i) a sequence of two 1st order transitions $\mathrm{NO} \rightarrow \mathrm{PS}(\mathrm{CB} / \mathrm{NO}) \rightarrow \mathrm{CB}$ (which was discussed in Sect. 2A); (ii) sequences of three 1st order transitions: $\mathrm{NO} \rightarrow \mathrm{PS}(\mathrm{CL} / \mathrm{NO}) \rightarrow \mathrm{PS}(\mathrm{CB} / \mathrm{CL}) \rightarrow \mathrm{CL}$, and $\mathrm{NO} \rightarrow$ $\mathrm{PS}(\mathrm{CL} / \mathrm{NO}) \rightarrow \mathrm{PS}(\mathrm{CB} / \mathrm{CL}) \rightarrow \mathrm{CB}$; (iii) a sequence of four 1st order transitions $\mathrm{NO} \rightarrow \mathrm{PS}(\mathrm{CL} / \mathrm{NO}) \rightarrow$ $\mathrm{PS}(\mathrm{CB} / \mathrm{CL}) \rightarrow \mathrm{CB} \rightarrow \mathrm{CL}$.

More detailed discussion of the case of PS involving $\mathrm{CB}$ and $\mathrm{CL}$ CO for arbitrary concentration and $T \neq 0$ will be given elsewhere.

\section{Conclusions}

We have presented results for the $t-W$ and the MC models considering both the homogeneous phases (NO,
$\mathrm{CL}, \mathrm{CB})$ and the phase separated states $[\mathrm{PS}(\mathrm{CL} / \mathrm{NO})$, $\mathrm{PS}(\mathrm{CB} / \mathrm{NO}), \mathrm{PS}(\mathrm{CL} / \mathrm{CB})]$, for $d=2 \mathrm{SQ}$, non-frustrated $\left(t_{2}=0\right)$ and frustrated $\left(t_{2} \neq 0\right)$ lattices, at half fillings. We have found various possible sequences of transitions.

We compared results for the two cases (i) involving CB CO only and (ii) involving two types of CO: CB and CL. Generally we may conclude that the phase diagrams at half-filling are similar for both cases in the absence of frustration while they are substantially different for large $t_{2}$, where the existence of the CL CO states considerably modifies the GS diagram structure. One can also conclude that at half filling the ground state diagram structures of both models ( $W$ vs. $b$ and $G$ vs. $b$ ) are qualitatively similar for any considered values of $b$.

\section{References}

[1] R. Micnas, J. Ranninger, S. Robaszkiewicz, Rev. Mod. Phys. 62, 113 (1990).

[2] S. Robaszkiewicz, G. Pawłowski, Acta Phys. Pol. A 90, 569 (1996).

[3] W. Czart, S. Robaszkiewicz, B. Tobijaszewska, Acta Phys. Pol. A 114, 73 (2008).

[4] S. Robaszkiewicz, T. Kostyrko, Physica 112B, 389 (1982); 121B, 134 (1983).

[5] P. Fulde, P. Thalmeir, G. Zwicknagel, Solid State Phys. 60, 1 (2006).

[6] K. Rościszewski, A.M. Oleś, J. Phys., Condens. Matter 15, 8363 (2003).

[7] H. Seo, J. Merino, H. Yoshioka, M. Ogata, J. Phys. Soc. Jpn. 75, 51009 (2006).

[8] W. Czart, P.R. Grzybowski, S. Robaszkiewicz, Acta Phys. Pol. A 118, 369 (2010).

[9] T. Kostyrko, R. Micnas, Phys. Rev. B 46, 11025 (1992).

[10] G.S. Uhrig, R. Vlaming, Ann. Phys. 4, 778 (1995). 